\title{
Reporting of compliance in randomised controlled trials investigating the effect of maternal nutritional supplementation on infant outcomes
}

\author{
Sarah Kehoe ${ }^{1}$, Purvi Chheda ${ }^{2}$, Siraj Ameen Sahariah ${ }^{2}$, Janis Baird ${ }^{1}$ and Caroline Fall ${ }^{1}$ \\ ${ }^{1}$ University of Southampton, Southampton, UK and ${ }^{2}$ Centre for Study of Social Change, Mumbai, India
}

The Consolidated Standards of Reporting Trials (CONSORT) group comprises medical journal editors, epidemiologists and clinical trialists and was established in 1996 with the aim of developing scales to assess the quality of randomised controlled trial (RCT) reports. The group have since developed several evidence-based initiatives to improve the reporting of RCT. One of these initiatives is the Revised CONSORT Statement ${ }^{(1)}$ that is made up of a checklist and participant flow diagram to be utilised by authors when reporting trials.

The participant flow diagram specifies the data that should be reported to describe the progress of participants through a trial. It is recommended that the number of participants that 'received' the treatment and the number that 'discontinued' treatment be stated. The flow chart does not, however, specify that the percentage of participants complying with the treatment protocol be reported. This information is particularly important in long-term supplementation trials where poor compliance may influence the way in which study findings are interpreted.

The aim of the present study was to investigate the adequacy of reporting of: (1) participant flow data as recommended by CONSORT; (2) participant compliance data (using criteria developed for the purpose of the current study) from RCT that studied the effect of maternal nutritional supplementation during pregnancy on neonatal and infant outcomes including birth weight, mortality and nutritional status.

A literature search of MEDLINE, EMBASE, CINAHL and AMED databases was carried out using the following combination of keywords: one of thirty-one nutrient terms (e.g. Ca) + pregnancy or birth + trial. Papers were included if they were published $\geq 1$ year after the CONSORT Statement was released and reported effects of maternal nutritional interventions before or during pregnancy on infant outcomes such as birth weight or growth. Papers reporting effects on 'maternal' outcomes such as eclampsia were excluded. All papers were systematically reviewed by two reviewers and categorised according to whether CONSORT participant flow data and compliance data were reported. Any discrepancies were discussed with a third reviewer and consensus reached.

Outcome measures were the presentation of the following data within the text or tables of the paper: participant flow data recommended in the CONSORT Statement; participant compliance data according to 'compliance' criteria: method of assessing participant compliance, compliance rate (proportion or absolute number of compliers), differences in compliance rate between treatment arms, researcher input aimed at maximising compliance.

Fifty-eight papers met inclusion criteria. Approximately one-third ( $n$ 18) did not provide details of how compliance was assessed. Approximately half $(n 31)$ reported compliance rate numerically. Differences in compliance values between treatment arms of the study were reported in $48 \%$ of papers $(n 28)$ and $83 \%(n 48)$ gave no information on whether the study protocol included attempts to maximise participant compliance. On average, papers reported 74 (SD 18) \% of data recommended by CONSORT.

Inadequate reporting of participant compliance with the study intervention frequently occurs in journal articles. The reporting of 'false negative' results from RCT with poor participant compliance could erroneously influence policy and further research concerned with nutritional supplementation for women of child-bearing age. Changes to the CONSORT recommendations may improve the quality of compliance reporting in supplementation trials.

1. Moher D, Schulz KF \& Altman DG (2001) Lancet 357, 1191-1194. 